\title{
Mecanobiología de los huesos maxilares. III. Regeneración ósea
}

\author{
Cano-Sánchez J*, Campo-Trapero J**, Restoy-Lozano A***, \\ Bascones-Martínez A****
}

\section{RESUMEN}

La mecanobiología ósea se encarga de la interacción entre las señales mecánicas y los mecanismos moleculares en las células del tejido óseo. Las cargas mecánicas actúan sobre las células que se encuentran en el nuevo foco de fractura y sobre las células incluidas en la matriz extracelular, por lo que la influencia mecánica actúa sobre la regeneración y sobre el remodelado, que se encuentran interrelacionados. El presente artículo intenta establecer la relación entre la carga y la expresión molecular durante la regeneración ósea. También, se realiza una revisión de los estudios de distracción alveolar histogénica y de carga en los implantes dentales en la fase de regeneración ósea.

Palabras clave: Mecanobiología, hueso, implantes.

\section{SUMMARY}

Bone mechanobiology deals with connection between mechanical signals and molecular events in cells and bone tissue. Mechanic loading has an influence both over cells in fracture callus and cells included into the extracellular matrix, thus mechanical forces have an interconnection between regeneration and remodelling. This paper tries to show the connection between loading and molecular expression during bone regeneration. Studies about alveolar distraction and dental implants under immediate loading are also reviewed.

Key words: Mechanobiology, bone, implants.

Fecha de recepción: Abril 2007.

Aceptado para publicación: Diciembre 2007.

* Profesor asociado. Facultad de Odontología UCM.

** Profesor contratado doctor. Facultad de Odontología UCM.

*** Medico adjunto del Servicio de Cirugía Oral y Maxilofacial. Hospital Príncipe de Asturias. Alcalá de Henares.

**** Catedrático de Medicina Bucal y Periodoncia. Facultad de Odontología UCM.

Cano-Sánchez J, Campo-Trapero J, Restoy-Lozano A, Bascones-Martínez A. Mecanobiología de los huesos maxilares. III. Regeneración ósea. Av. Odontoestomatol 2008; 24 (3): 227-237.

\section{INTRODUCCIÓN}

La aplicación de la carga puede tener diferentes resultados y mecanismos dependiendo si se aplica en un proceso de curación o regeneración ósea (formación de nuevo hueso inmaduro) en compara- ción con un proceso de remodelado ósea (transformación de un hueso inmaduro por un hueso maduro o reemplazo de un hueso maduro dañado por otro hueso maduro) (1). En el caso de la aplicación de carga sobre un escenario de curación ósea ya existen modelos probados durante años, 
como es el caso de la distracción histogénica o la aplicación de carga inmediata sobre los implantes dentales.

La distracción histogénica se define como la capacidad para desarrollar hueso neoformado y tejidos blandos adyacentes, entre dos fragmentos óseos que previamente han sido separados por osteotomía y se les va distanciando gradualmente sin interrumpir el aporte sanguíneo $(2,3)$.

La aplicación de carga inmediata sobre implantes dentales se ha basado hasta ahora en la existencia de una estabilidad primaria del implante suficiente para realizar esa carga, sin considerar el tipo, magnitud y duración de carga que podrían recibir tales implantes. De este modo, la carga inmediata que se está aplicando actualmente sobre los implantes en curación perimplantaria es completamente descontrolada y dependiente de los movimientos masticatorios del paciente.

Hay que tener en cuenta que los factores mecánicos que actúan sobre un ambiente de regeneración ósea, actúan a la vez sobre el hueso sano maduro adyacente que ha sufrido el trauma (ya sea traumatismo u osteotomía quirúrgica). Es decir, las cargas mecánicas actúan sobre las células que se encuentran en el nuevo foco de fractura (células mesenquimales indiferenciadas y osteoblastos en diferenciación) y sobre las células incluidas en la matriz extracelular (osteocitos). En este sentido, existiría una influencia mecánica sobre la regeneración y una influencia mecánica sobre el remodelado, que se encuentran interrelacionados.

En una publicación anterior se describieron los eventos de mecanobiología que intervienen en el remodelado óseo. En el presente artículo se describen tales eventos cuando se desarrollan en la fase inicial de regeneración. Para ello se ha realizado una revisión de la literatura de los diferentes estudios de mecanobiología ósea en general y la que estudia su comportamiento en los huesos maxilares. El presente artículo intenta establecer la relación entre la carga y la expresión molecular durante la regeneración ósea. También, se realiza una revisión de los estudios de distracción alveolar histogénica y de carga en los implantes dentales en la fase de regeneración ósea.

\section{MECANISMO CELULAR Y MOLECULAR DE LA REGENERACIÓN ÓSEA SOMETIDA A CARGA}

Se ha descrito que existen muchos procesos biológicos, como la proliferación, la diferenciación, la migración, la producción de proteínas, e incluso la apoptosis que están influenciadas por los cambios de la forma celular y la integridad estructural (4). En ocasiones la diferenciación celular puede asimilarse a la expresión de proteínas, ya que ciertas proteínas (p. ej.: Osteocalcina, OCN) son marcadores de la diferenciación celular (p. ej.: osteoblastos).

La diferenciación de las células mesenquimales en la estirpe osteoblástica, se ve influenciada por factores biomecánicos, de tal manera que en traumatología se ha observado que fuerzas compresivas moderadas favorecen la regeneración del callo de fractura, vía osificación endocondral (5). Al igual que en la distracción (donde existen fuerzas de tensión), si las fuerzas son excesivas y si además no hay suficiente aporte vascular, la diferenciación va a ser fibrosa o fibrocartilaginosa y no osteogénica. Las magnitudes o el límite de esas fuerzas compresivas todavía no están bien definidas. Se ha observado con elementos finitos que en la distribución del estrés tanto en compresión cíclica de fractura como en distracción, existe una mayor deformación en las partes externas de la cámara de fractura (6), lo que explicaría el resultado clínico de formación ósea en forma de "reloj de arena" ya que en esa zona externa hay un tipo de deformación (distorsional) que dificultaría la diferenciación osteoblástica.

El efecto del estrés biomecánico sobre las células puede ser de tipo volumétrico o dilatacional (estrés hidrostático), en el que no hay modificación de la morfología celular sino solo una exudación de liquido intracelular con modificación de su volumen, debido a aplicación de fuerzas tensionales o compresivas moderadas o discontinuas; o bien un estrés de tipo distorsional (estrés de cizalla octaédrica) en el que sí existe una deformación morfológica de la célula, por aplicación de fuerzas tangenciales o de cizalladura, o por unas fuerzas tensionales o compresivas elevadas y/o continuas. Parece que las fuerzas distorsionales alterarían la estructura del citoesqueleto e inducirían la diferenciación mesenquimal hacia el linaje fibroblástico-cartilaginoso, mientras 
que las volumétricas o hidrostáticas favorecerían y acelerarían la diferenciación hacia el linaje osteoblástico (6).

Diferentes estudios in vitro han intentado resolver la respuesta de las células a las cargas. Con este objetivo se han realizado estudios con diversas estrategias experimentales. Se ha desarrollado colocación de células en placas de cultivo y aplicación de un estrés de cizallamiento mediante un fluido. Con este método Klein-Nulen y cols. observaron que las zonas estimuladas presentaban mayor liberación de Óxido Nítrico (NO) y Prostaglandina E2 (PGE2). (7)

Se ha desarrollado estimulación de células individuales mediante Microscopio de Fuerza atómica (AFM, por Atomic Force Microoscope). Se ha observado que la presión individual de osteoblastos origina una mayor producción de $\mathrm{Ca}^{2+}(8)$. También se ha realizado aspiración de células mediante micropipetas para observar su grado de deformación y así crear posteriormente modelos con elementos finitos (9).

En la distracción histogénica, la fase de activación (de distracción) se suele realizar durante una o dos semanas y la tracción modifica el normal desarrollo del proceso de regeneración. Se forma un "microambiente dinámico" que conlleva la formación de tejido de manera paralela al vector de tracción. En el proceso de distracción hay un aumento y prolongación de la angiogénesis y un incremento en la proliferación de células fusiformes similares a fibroblastos (spindle-shaped fibroblast-like cells) que presenta una variación fenotípica (hipertrofia de los filamentos intermedios citosólicos con apariencia alargada de la célula). Este tipo de células fusiformes se sitúan periféricamente y a lo largo de los vasos produciendo más colágeno de manera paralela al vector de distracción y ultraestructuralmente se caracterizan por un aumento del retículo endoplásmico en el citoplasma y aumento del nucleolo en el núcleo (10).

El estudio inmunohistoquímico realizado por Ilizarov $(2,3)$ demostró que en la fase de distracción existe un aumento de la fosfatasa alcalina, aumento del ácido pirúvico, y aumento del ácido láctico (productos del metabolismo enzimático). Parece que esa tensión moderada y controlada que ejerce el distractor en el tejido de granulación produciría una mayor diferenciación de las MSCs (por Mesenchymal Stew Cells) en osteoblastos, y por otro lado favorecería la mayor producción de proteínas óseas por parte de los osteoblastos $(6,10)$.

Los factores biomecánicos no solo van a influir en la producción de proteínas exclusivas de un tipo de diferenciación celular (p. ej.; OCN en osteoblastos) sino también en la producción de otro tipo proteínas óseas importantes en la biología ósea. Parece que la aplicación de fuerzas hidrostáticas moderadas (tensionales o compresivas) induciría la mayor deposición de estas proteínas, lo cual sería el fundamento de la más rápida formación ósea en los procesos de distracción histogénica (6). También sería el fundamento de aplicación clínica de la carga inmediata o precoz. El mecanismo molecular exacto y el tipo y cantidad de fuerzas a aplicar son todavía desconocidas, aunque en distracción hay unos valores de tensión o distracción más favorables (aproximadamente $1 \mathrm{~mm}$ de tensión al día), ya establecidos pero sin una correlación molecular.

Mediante técnicas de inmunohistoquímica tras elongación de mandíbula de ratas se ha observado la sobreexpresión de TGF- $\beta 1$ (por Transforming Growth Factor $\beta 1$ ) tanto en fases de latencia, de distracción, como hasta la cuarta semana del periodo de consolidación volviendo después a niveles basales (11). Este factor parece que tiene, entre otras, una función de promoción de la angiogénesis y de producción de colágeno tipo I. La expresión de OCN se incrementa a partir del cuarto día de latencia hasta alcanzar niveles de un incremento del $70 \%$ a las del hueso normal a las cuatro semanas de consolidación. También se ha visto decrecimiento de OCN y ONN (Osteonectina) cuando se aplican tensiones hiperfisiológicas en elongación de mandíbula de conejo. Estos autores establecen los $3.000 \mu \varepsilon$ como el límite a partir del cual los microtraumas sobrepasarían la capacidad de reparación. La ventana entre 2.000 y $3.000 \mu \varepsilon$ sería la apropiada para mejorar la regeneración ósea (12).

El colágeno tipo I, como enucleador de los cristales de hidroxiapatita es muy sensible a la cantidad de 
carga que recibe. De esta manera, la microarquitectura del colágeno es alterada, produciéndose un desensamblaje de las fibras y se produce menor deposición de hidroxiapatita cuando es sometido a cargas elevadas $(>20.000 \mu \varepsilon)(13)$.

Utilizando fémur de rata, Sato y cols observaron en fases finales de distracción que la OCN se encontraba no solo en los osteoblastos diferenciados sino también en los preosteoblastos y los fibroblastos fusiformes (14). La OPN (por Osteopontina) se encontraba exclusivamente en los preosteoblastos y osteoblastos diferenciados mientras que la ONN aparecía en todas las células de la zona. Mediante Northern Blot observaron que tanto la OPN, OCN y ONN no daban señal en el tejido óseo intacto, empezaba a dar señal a los 7 días de las muestras con osteotomía y luego disminuía, mientras que en las muestras distraídas aumentaba su señal mientras duraba la distracción (21 días) y luego disminuía en la consolidación.

En tibias de ovejas se observó también que los niveles séricos de OCN se incrementaban un $15 \%$ durante la fase de distracción, pero más de un $100 \%$ a los 44 días de la fase de consolidación, a partir de lo cuales se estabilizan los niveles (15). Esto demuestra el incremento de la actividad osteoblástica tras la aplicación de tensión.

La expresión de BMP-2 (por Bone Morphogenetic Protein-2), BMP-4, y BMP-7, también se incrementa durante la fase distracción en relación con la fase de latencia y va desapareciendo durante la fase de consolidación. Esto se ha visto en tibias de conejo donde solo las MSCs y los preosteoblastos evidenciaron estas moléculas pero no los osteoblastos (16).

En cualquier caso, hoy en día es desconocido si la aceleración en la maduración del callo óseo que se produce en la distracción es debido a una rápida maduración de la malla de colágeno y por tanto una mayor superficie para el depósito de osteoide, o bien es debido a los efectos estimuladores que mejoran la proliferación de osteoblastos. En otras palabras, no se sabe si es debido a un incremento en el número de osteoblastos reclutados, o bien es debido al aumento de la actividad de síntesis de cada célula individual, o bien es debido a ambos factores a la vez.

\section{MECANOBIOLOGÍA DE HUESO EN REGENERACIÓN POR DISTRACCIÓN}

Desde que Ilizarov reintrodujera el fenómeno de la distracción en la terapéutica ortopédica mediante un método científico contrastado, han aparecido nuevas aplicaciones en el resto de la economía ósea del organismo $(2,3)$. Una de las localizaciones anatómicas donde se ha introducido y se está investigando esta técnica es la regeneración de la cresta alveolar, tanto en la mandíbula como en el maxilar. El procedimiento se ha extendido desde su primera aplicación clínica realizada por Chin y cols. en 1996 (17), tanto en estudios experimentales (18-20), como en estudios clínicos $(21,22)$.

Los estudios experimentales siguen siendo escasos en distracción alveolar. Block y cols (2000) realizaron un estudio experimental en mandíbula de perros utilizando un prototipo de distractor extraóseo (18). El estudio radiológico reveló que a las 6 semanas después de terminar la distracción aumentaba la densidad del gap de distracción. La evaluación histológica al año de carga fue similar en todas las muestras, no existiendo diferencias del hueso trabecular entre zonas distraídas y no distraídas. En relación con el espesor del hueso cortical si se observaron diferencias entre las zonas distraídas y no distraídas, y entre hueso labial y lingual (menor y mayor respectivamente) pero no en relación con los 2 tipos de implantes.

Oda y cols (2000) realizan un estudio en mandíbula de 6 perros utilizando un prototipo de distractor intraóseo. Se realizó un estudio radiológico con valoraciones a las $0,2,4,8$ y 12 semanas postdistracción, observando radiolucidez del gap de distracción a las 4 semanas, apareciendo una densidad radiológica importante a las 8 semanas que no se diferenciaba de la observada a las 12 semanas. Se realizó también un estudio histomorfométrico donde se vio unos valores de contacto hueso-implante menores a las 8 semanas que a las 12 semanas (19).

Gaggl y cols (2000) realizaron un estudio de distracción alveolar en mandíbulas de ovejas, en el que mediante el distractor-implante alveolar DISSIS observaron que a partir del 3 mes de consolidación aparecía mayor radiodensidad y presencia de hueso 
laminar en el centro del gap que no aparecía al mes y a los dos meses de consolidación (20). A los 6 meses de consolidación se observó una aposición hueso-distractor del $70-80 \%$ sin que se considerara que había terminado la reestructuración ósea. Establecieron que a partir del 3 mes de consolidación había una estabilidad del distractor-implante suficiente (valores de periotest < 3) y se podría iniciar el tratamiento prostodóncico.

Nosaka y cols (2002) también describen un prototipo de distractor alveolar horizontal utilizando como biomodelo la mandíbula del perro beagle (23). En el estudio radiológico se observó radiolucidez de la zona a los 12 días de consolidación, momento en el que se colocaron los implantes. A las 8 semanas después de colocar los implantes existía un radiodensidad de la cámara y ligera reabsorción del hueso transportado. A las 12 semanas después de colocar los implantes se observaba hueso laminar en contacto con el implante pero en menor cantidad que a las 24 semanas. Consideran que las 24 semanas sería el momento propicio para iniciar la carga prostodóncica, ya que en este momento observaron proporciones similares de BIC en comparación a los implantes control. En ese momento vieron que el fragmento transportado se había reabsorbido totalmente.

Se ha comparado en mandíbula de perros el procedimiento de distracción alveolar con los injertos en onlay (24). Se observaron varios parámetros morfométricos que indicaban un mayor índice de remodelado en el grupo de distracción a los cinco meses de consolidación. Esto parece indicar una aceleración del proceso total de regeneración ósea.

Nuestro equipo de investigación realizó un estudio experimental en mandíbula de perro mediante un prototipo de distractor (25). Se valoró mediante histomorfometría el hueso regenerado tras 4 y 8 semanas de periodo de consolidación. Se observó que a las 4 semanas existía un predominio de hueso inmaduro reticular mientras que a las 8 semanas un predominio de hueso inmaduro de fibras paralelas, pero sin presencia de hueso maduro laminar. Se observó también una mayor densidad ósea en la zona lingual que en la zona bucal. Se estableció la hipótesis de una colocación temprana de los implantes para evitar la reabsorción del hueso transportado (25).
Los estudios clínicos con distracción alveolar se iniciaron con series de pacientes pequeñas y con una amplia variedad de diseños de distractor. En los últimos 5 años se ha impuesto la utilización de los distractores extraóseos sobre los distractores intraóseos y los implante-distractor, debido a que presentan menor tasa de complicaciones. De este modo, se han multiplicado los trabajos clínicos en distracción alveolar presentando mayores tamaños muestrales.

Uno de los factores condicionantes de la distracción que requiere más interés es el del vector de distracción. En los casos de distracción maxilar se ha recomendado el acceso palatino de tal manera que el hueso transportado queda unido al pedículo mucoperióstico vestibular que permite un mejor control del vector de distracción (26). En otras ocasiones el vector de distracción se ha intentado controlar mediante el propio diseño del distractor. Robiony y cols diseñan un distractor bidireccional que controla el movimiento vestibulolingual además del vertical (27). Ya sea por el vector de distracción o por otros factores condicionantes se ha demostrado un alto índice de defectos en la cresta ósea después de la regeneración por distracción. De esta manera, usando un distractor intraóseo, se ha observado una mayor proporción de defectos cuando se incrementa la altura inicial en un $25 \%$ (28). Se esta proponiendo modificaciones en la fase de distracción para evitar la frecuente cresta en "reloj de arena". Para ello se proponen protocolos de "masaje del callo" o "bombeo del callo" donde se intercala tensión con compresión (29).

\section{MECANOBIOLOGÍA DEL HUESO PERIMPLANTARIO EN REGENERACIÓN}

La característica común que se observa en el espacio entre el hueso y un implante al colocar este último, es la aparición de un coágulo sanguíneo. Si el implante permanece estable, ese espacio será rellenado por nuevo hueso tras un proceso de osteogénesis intramembranosa. El proceso intramembranoso se caracteriza por una secuencia de pasos que incluye formación del coágulo, angiogénesis, reclutamiento y migración de MSCs, diferenciación osteoblástica, formación de hueso reticular, compac- 
tación del hueso reticular formando hueso de fibras paralelas y finalmente formación de hueso laminar tras un proceso de remodelado (30).

Hay que tener en cuenta que el remodelado óseo es activado inmediatamente o días después de que se produzca el trauma quirúrgico en el hueso. La diferenciación de los osteoclastos a partir de las células madre hematopoyéticas va a iniciar la reabsorción y renovación de la capa de hueso necrótico (aprox. 1 $\mathrm{mm}$ ) que se ha producido tras el trauma inicial. Parece que en el hueso trabecular el espesor de esta zona necrótica es menor que en el hueso cortical, por lo que necesitaría un proceso de remodelado más corto y la regeneración perimplantaria sería más rápida. Se ha observado en hueso cortical que para reparar esa zona necrosada se necesita un ciclo de remodelado (sigma) que tiene una duración de 3 meses en el perro y de 4-5 meses en el humano (31).

La interfase hueso-implante fue descrita inicialmente como una zona rica en proteoglicanos y glicosaminoglicanos de aproximadamente $200 \AA$ (aprox. 200 $\mathrm{nm}$ ), en estos estudios iniciales se descalcificaba el hueso anulando su fase mineralizada. Estudios mediante inmunocitoquímica de alta resolución, demostraron una capa electrodensa relativamente mineralizada formada por proteínas de la matriz no colagénicas como la OPN, OCN y BSP (por Bone Sialoprotein) que tienen una capacidad de adhesión celular y de enucleación mineral (32).

Davies describió la cascada de lo que denomina la "formación de nuevo hueso" en la curación perimplantaria en 4 fases: Adsorción de proteínas no-colagénicas en la superficie implantaria; mineralización de esas proteínas; crecimiento de los cristales; uniónrecubrimiento a esa matriz de interfase de una matriz de colágeno (33). De esta manera establece que la interfase hueso-implante sería una estructura sin red colagénica (línea de cementación) como ya describiera Weindenreich en 1930, que al microscopio electrónico presentaría un aspecto globular por las enucleaciones de los cristales minerales. Esta línea de cementación tendría un espesor aproximado de $0,5 \mu \mathrm{m}$, electrodensa al microscópico electrónico de transmisión. Estudios in vitro demuestran que su composición es formada por componentes orgáni- cos e inorgánicos: los orgánicos son en su mayoría proteoglicanos y glicoproteínas (en su mayoría OPN), y la parte inorgánica son fosfatos cálcicos (en su mayoría hidroxiapatita).

En las superficies de titanio parece que los iones de $\mathrm{Ca}^{2+}$ que precipitan en las proteínas de adhesión de la línea de cementación se unirían a el $\mathrm{TiO}_{2}$ que tiene carga negativa, obteniéndose una unión química débil electrostática (34). En los recubrimientos de $\mathrm{HA}$ al existir mayor proporción de compuestos de calcio presentes en su superficie, habría mayor unión con esas proteínas a través del calcio que tienen precipitado, y de una manera químicamente mas fuerte.

Diversos estudios han determinado la similitud entre el hueso que se forma alrededor de los implantes y las líneas de cementación del hueso normal, aunque con un espesor más grueso. Estas líneas de cementación del hueso normal no tiene una ultraestructura bien conocida, en relación a las cantidades exactas que tienen de colágeno, mucopolisacáridos, glicoproteínas y mineral, aunque se establece que es un tejido hipomineralizado con una resistencia biomecánica inferior al hueso mineralizado. En varios estudios de resistencia a la tensión se han observado valores en esta línea de cementación menores a 4 Mpa (incluso con los mejores resultados observados con los recubrimientos de hidroxiapatita) que es muy inferior a los valores del hueso cortical normal mineralizado que se encuentra entre 100-150 Mpa. Y tampoco se acerca a los valores de resistencia al cizallamiento del hueso mineralizado que es de 68 Mpa (35).

Se especula que las células preosteogénicas (que no han expresado totalmente el fenotipo osteoblástico) no producen colágeno, ni BSP, pero si OPN (que tiene una capacidad inhibitoria para la formación de cristales de hidroxiapatita) y son las encargadas de generar las líneas o superficies de inversión (donde se invierte la reabsorción en formación), mientras que los osteoblastos totalmente diferenciados si producen colágeno y son los encargados de producir las líneas o superficies de parada. Parece que la línea de cementación en los implantes se asimila más a las líneas de inversión, por lo que parece que la producirían células precursoras que 
todavía no expresarían la BSP, y por tanto con incapacidad de enucleación mineral y de formación de colágeno (36).

¿Cuál es el límite de micromovimiento que toleran los tejidos en regeneración alrededor del implante sin que se produzca la formación de tejido fibroso? Se ha estimado que con las superficies rugosas se podrían tolerar movimientos entre $50-150 \mu \mathrm{m}$ incluso inmediatamente después de la cirugía, aunque este rango no se ha evidenciado científicamente (37). Saber si se va sobrepasar ese valor desconocido cuando se aplique carga inmediata o precoz no se ha descrito con los medios diagnósticos y terapéuticos actuales, pero parece que va a depender del diseño y superficie del implante, calidad y cantidad ósea, el tipo de carga que se va a ejercer, y la situación estratégica de los implantes en la planificación prostodóncica. Parece que sobrepasar el umbral del micromovimiento (todavía no definido) podrían dañar la red de fibrina y los nuevos vasos que se están formando, así como la diferenciación de las MSCs hacia osteoblastos (35).

En este sentido hay autores como Misch y cols (38) que creen que el rango de carga ideal para los implantes dentales es la que se encuentra en el rango de carga fisiológica normal (1.000-1.500 $\mu \varepsilon$ ) descrita por Frost (39). Según estos autores en ese rango se facilitaría un hueso más maduro y resistente en la interfase hueso-implante, mientras que en rangos superiores (sobrecarga moderada: 1.500-4.000 $\mu \varepsilon)$ el hueso tendría un índice de recambio superior, menor grado de mineralización y menor organización del mismo. Estos autores establecen la hipótesis de que el hueso inmaduro no es adecuado para soportar las cargas oclusales aludiendo que tendría un mayor riesgo de microfracturas, debido a que se incrementa la diferencia de modulo de elasticidad entre el implante y el hueso perimplantario inmaduro.

Teniendo en cuenta estas hipótesis de Misch, se podrían establecer las siguientes preguntas:

a) ċen que rango de la clasificación de Frost se encuentran las cargas masticatorias a las que se someten los implantes en carga inmediata/pre$\mathrm{COZ}$ ? b) ces realmente el hueso inmaduro (reticular y de fibras paralelas) tan poco resistente y tan peligroso para resistir las cargas?

Hay que tener en cuenta que la expresión molecular celular difiere según las diferentes localizaciones anatómicas y las diferentes condiciones de carga. En este sentido hay que distinguir entre los resultados obtenidos en el hueso perimplantario dentro de un hueso maxilar o en huesos largos. Por otro lado, se debe diferenciar entre los resultados obtenidos en el hueso perimplantario tras la aplicación de carga controlada, es decir con unos valores cuantificables, y tras la aplicación de una carga descontrolada, es decir tras ser sometido a las cargas masticatorias. Teniendo en cuenta que el tiempo total de "sigma" en hueso cortical en conejo es de 6 semanas (40), en el perro es de 3 meses, y en el humano de 4-5 meses (41), y que la carga precoz en humanos se ha establecido dentro de los 3 primeros meses (42), se considera que la carga precoz en conejos sería de aproximadamente menos de 1 mes, y en perros de menos de 2 meses.

En la mayoría de estudios experimentales y clínicos (43-46) se han aplicado protocolos de carga para observar la biología ósea resultante durante la fase de remodelación, es decir tras esperar la regeneración previa de un hueso inmaduro perimplantario.

Algunos autores consideran que los implantes colocados en el área retromolar mandibular pueden soportar una carga ortodóncica inmediata $(<5 \mathrm{~N})$ sin observar complicaciones clínicas en dichos implantes (47). También se han descrito numerosos estudios clínicos y experimentales donde se aplica una carga descontrolada (carga masticatoria inmediata y/o precoz) sobre los implantes en pleno proceso de curación perimplantaria. Sin embargo, no se han descrito estudios histológicos que describan los procesos biológicos que acontecen en el hueso perimplantario sometido a carga controlada durante la fase de curación/regeneración o en huesos de baja densidad.

Duyck y cols diseñaron una cámara de crecimiento óseo que permite observar la regeneración ósea perimplantaria y al mismo tiempo someter al implante a carga axial (48). Utilizando tibias de conejo aplicaron una carga axial dinámica ( $50 \mu \mathrm{m}$ de desplazamiento axial) durante 4 semanas (800 ciclos/día, 2 veces por 
semana, a una frecuencia de $1 \mathrm{~Hz}$ ) de manera inmediata posterior a la implantación. Observaron en los implantes cargados una BIC $(13,16 \%)$ muy superior a la de los implantes no cargados $(0,65 \%)$.

\section{CONCLUSIONES}

- La carga mecánica origina una expresión determinada de moléculas en el foco de curación debido a la deformación celular. La distorsión volumétrica controlada de la célula produce una mayor producción de OCN, de TGF- $\beta 1$, colágeno tipo I, y diferentes formas de BMP.

- Los estudios clínicos y experimentales por distracción son todavía limitados y con seguimientos cortos.

- Los estudios de carga inmediata sobre implantes dentales presentan carencias en describir el fenómeno molecular que acontece en el hueso perimplantario. Los límites objetivos de deformación durante las cargas masticatorias son todavía desconocidas.

\section{BIBLIOGRAFIA}

1. Clark PA, Rodriguez A, Summer DR, Hussain MA, Mao JJ. Modulation of bone ingrowth of rabbit femur titanium implants by in vivo axial micromechanical loading. J Appl Physiol 2005;98: 1922-9.

2. Ilizarov G. The tension-Stress effect on the genesis and growth of tissues. Part I.The influence of stability of fixation and soft tissue preservation. Clin Orthop Rel Res 1989;238:249-81.

3. Ilizarov G. The tension-Stress effect on the genesis and growth of tissues. Part II. The influence of the rate and frequency of distraction. Clin Orthop Rel Res 1989b;239:263-85.

4. Lim CT, Zhou EH, Quek ST. Mechanical models for living cells. A review. J Biomech 2006;39:195216.

5. Matsushita T, Kurokawa T. Comparison of cyclic compression, cyclic distraction and rigid fixation: bone healing in rabbits. Acta Orthop Scand 1998; 69:95-8.

6. Carter DR, Polefka EG, Beaupre GS. Mechanical influences on skeletal regeneration and bone resorption. En Davies JE, eds; Bone engineering; Toronto: em squared 2000:358-80.

7. Klein-Nulend J, Semeins CM, Burger EH, Van der Plas A, Ajubi NE, Nijweide PJ: Response of isolated osteocytes to mechanical loading in vitro, In Odgaard A, Weinans H (Eds.). Bone Structure and Remodelling, Singapore: World Scientific; 1996:37-49

8. Charras GT, Horton MA. Single cell mechanostransduction and its modulation analyzed by atomic force microscopy identation. Biophysics J 2002;82:2970-81.

9. Jones WR, Ting-Beall HP, Lee GM, Kelley SS, Hochmuth RM, Guilak F. Alteration in the Young's modulus and volumetric properties of chondrocytes isolated from normal and osteoarthritic human cartilage. J Biomech 1999; 32:119-27.

10. Samchukov ML, Cope JB, Cherkashin AM. Biological basis of new bone formation under the influence of tension stress. En Samchukov ML, Cope JB, Cherkasin AM (eds). Craniofacial distraction osteogenesis . St Louis:Mosby, 2001: 21-52.

11. Mehrara BJ, Rowe NM, Steinbrech DS, Steinbrech DS, Dudziak ME, Saadeh PB, McCarthy JG, Gittes GK, Longaker MT. Rat mandibular distraction osteogenesis: II. Molecular analysis of transforming growth factor beta- 1 and osteocalcin gene expression. Plast Reconst Surg 1999;103(2):536-47.

12. Meyer U, Meyer T, Vosshans J, Joos U. Decreased expresion of osteocalcin and osteonectin in relation to high strains and decreased mineralization in mandibular distraction osteogenesis. J Craniomaxillofac Surg 1999;27: 222-7. 
13. Meyer U, Joos U, Kruse-Lösler B, Meyer T. Mechanically induced tissue response during distraction. En Samchukov ML, Cope JB, Cherkasin AM, eds. Craniofacial distraction osteogenesis. St Louis: Mosby, 2001:42-52.

14. Sato $M$, Yasui $N$, Nakase $T$, Kawahata $H$, Sugimoto M, Hirota S, Kitamura Y, Nomura S, Ochi T. Expresión of bone matrix proteins mRNA during distraction osteogenesis. J Bone Mineral Res 1998;13(8):1221-31.

15. Windhagen $\mathrm{H}$, Wite F, Hurschler C, Maciejewski O, Linnenberg D, Thorey F. Bone turnover during distraction osteogenesis in an experimental sheep model. Arch Orthop Trauma Surg 2002;122: 279-82.

16. Rauch F, Lauzier D, Croteau S, Travers R, Glorieux FH, Hamdy R. Temporal and spatial expression of bone morphogenetic protein-2, -4 , and -7 during distraction osteogenesis in rabbits. Bone 2000;26(6):611-7.

17. Chin M, Toth BA . Distraction osteogenesis in maxillofacial surgery using internal devicesReview of five cases. J Oral Maxilofac Surg 1996; 54:45-53.

18. Block MS, Gardiner D, Almerico B, Neal C. Loaded Hydroxylapatite-coated implants and uncoated titanium-threated implants in distracted dog alveolar ridges. Oral Surg Oral med Oral Pathol 2000;89(6):676-85.

19. Oda T, Sawaki Y, Ueda M. Experimental alveolar ridge augmentation by distraction osteogenesis using a simple device that permits secondary implant placement. Int J Oral Maxillofac Implants 2000;15:95-102.

20. Gaggl A, Schultes G, Regauer S, Karcher H. Healing process after alveolar ridge distraction in sheep. Oral Surg Oral Med Oral Pathol Oral Radiol End 2000;90(4):420-9.

21. Klug CN, Millesi-Schobel GA, Millesi M, Watzinger F, Ewers R. Preprosthetic vertical distraction osteogenesis of the mandible using and L-shaped osteotomy and titanium membranes for guided bone regeneration. J Oral Maxillofac Surg 2001; 59:1302-8.

22. Chiapasco M, Romeo E, Vogel G. Vertical distraction osteogenesis of edentulous ridges for improvement of oral implant positioning: A clinical report of preliminary results. Int $\mathrm{J}$ Oral Maxillofac Implants 2001;16(1):43-51.

23. Nosaka Y, Kitano S, Wada K, Komori T. Endosseous implants in horizontal alveolar ridge distraction osteogenesis. Int J Oral Maxillofac Imp 2002; 17:846-53.

24. Hodges NE, perry M, Mohamed W, Hallmon WW, Rees T, Opperman LA. Distraction osteogenesis versus autogenous onlay grafting. Part II: Biology of regenerate and onlay bone. Int $\mathrm{J}$ Oral Maxillofac Impl 2006; 21237-44.

25. Cano J, Campo J, Gonzalo JC, Bascones A. Consolidation period in alveolar distraction. A pilot histomorphometric study in mandible of beagle dog; Int J Oral Maxillofac Impl 2006;21: 380-91.

26. Garcia A, Somoza M, Gándara P, Saulacic N, Gandara JM. Palatal approach for maxillary alveolar distraction. J Oral Maxillofac Sur 2004; 62:795-8.

27. Robiony M, Toro C, Stucki-McCormick SU, Zerman N, Costa F, Polito M. The "FAD" (Floating Alveolar Device): A bidirectional distraction system for distraction osteogenesis of alveolar process. J Oral Maxillofac Surg 2004; 62:136-42.

28. Saulacic N, Somoza M, Gandara P, Garcia A. Bonde defect formation during implant placement following alveolar distraction. Int J Oral Maxillofac Imp 2007;22:47-52.

29. Mofid MM, Inoue N, Atabey A, marti G, Chao E, Manson PN, Vander Kolk CA. Callus stimulation in distraction osteogenesis. Plast Reconst Surg 2002;109:1621-19. 
30. Cano J, Martínez-González JM, Campo J, Martínez-González MJS, Donado A. Biología ósea perimplantaria. Periodoncia y Osteointegración 2002;12(5):345-56.

31. Roberts WE, Bone tissue interface. J Dent Educ 1988;52:804-9.

32. Nanci A, McCarthy Gf, Zalzal S Clockie CML, Warshawsky $\mathrm{H}$, McKee MD. Tissue response to titanium implants in the rat tibia: ultraestructural, immunicytochemical and lectin-cytochemical characterizacion of the bone-titanium interface. Cells Mat 1994;4:1-30.

33. Davies JE, Hosseini MM. Histodynamics of endosseous wound healing. En Davies JE, eds. Bone engineering. 2000. Em squared. Toronto: 1-14.

34. Ayukawa Y, Takeshita F, Inoue T, Yoshinari M, Shimono M, Suetsugu T, Tanaka T. An inmunoelectron microscopic localization of noncollagenous bone proteins(osteocalcin and osteopontin) at the bone-titanium interface of rat tibiae. J Biomed Mat Res 1998;41:111-9.

35. Brunski JB, Puleo D, Nancy A. Biomaterials and biomechanics of oral and maxillofacial implants: currents status and future developments. Int $\mathrm{J}$ Oral Maxillofac Impl 2000;15(1):15-46.

36. Hosseini MM, Sodek J, Franke RP, Davies JE. The structure and composition of the boneimplant interface. En Davies JE, eds. Bone engineering. 2000 a. em squared. Toronto:295304.

37. Szmukler-Moncler S, Piatelli A, Favero GA, Dubruille JH. Considerations preliminary to the application of early and immediate loading protocols in dental implantology. Clin Oral Impl Res 2000; 11:12-25.

38. Misch CE, Bidez MW, Sharawy M. A bioengineered implant for a predetermined bone cellular response to loading forces. A literature review and case report. J Periodontology 2001; 72 : 1276-86.
39. Frost HM. Wolff's law and bone's structural adaptations to mechanical usage: an overview for clinicians. The Angle Orthodontics 1994; 64: 175-88.

40. Roberts WE, Smith RK, Zilberman Y, Mozsary PG, Smith RS. Osseous adaptation to continuous loading of rigid endosseous implants. Am J Orthod 1984; 86(2):95-111.

41. Takahashi H, Norimatsu H, Watanabe G, Konno T, Inoue J, Fukuda M. The remodeling period (sigma) in canine and human trabecular bone. In Yoshitoshi U, Fujita T, eds. Calcium endocrinology. Tokio: Chugai Igaku co 1980: 13-31.

42. Cochran DL, Morton D, Weber HP. Consensus statements and recommended clinical procedures regarding loading protocols for endosseous dental implants. Int J Oral Maxillofac Imp 2004;19 suppl:109-13.

43. Gotfredsen K, Berglundh T, Lindhe J. Bone reactions adjacent to titanium implants subjected to static load. A study in the dog (I). Clin Oral Implants Res 2001a;12(1):1-8.

44. Akin-Nergiz N, Nergiz I, Schulz A, Arpak N, Niedermeier W. Reactions of peri-implant tissues to continous loading of osseointegrated implants. Am J Orthod Dent Orthop 1998;114 (3):292-8.

45. Melsen B, Lang NP. Biological reactions of alveolar bone to orthodontic loading of oral implants. Clin Oral Imp Res 2001;12:144-52.

46. Roberts WE, Marshall KJ, Mosary PG. Rigid endosseous implants utilized as anchorage to protract molars and close an atrophic extraction site. Angle Orthod 1990; 60:13552.

47. Roberts E. Anclaje ortodóncico con implantes oseintegrados: fisiología ósea, metabolismo y biomecánica. En Higuchi KW, eds. Aplicaciones ortodóncicas en implantes os- 
teintegrados. Caracas: AMOLCA; 2002: 16191.

48. Duyck J, De Cooman M, Puers R, Van Oosterwyck HV, Sloten JV, Naert I. A repeated sampling bone chamber methodology for the evaluation of tissue differentiation and bone adaptation around titanium implants under controlled mechanical conditions. J Biomech 2004; 37: 1819-22.

\section{CORRESPONDENCIA}

Dr. Jorge Cano Sánchez

Departamento de Medicina y Cirugía Bucofacial Facultad de Odontología- UCM

Plaza Ramon y Cajal, s/n 28040 Madrid

Email: jo.cano@wanadoo.es 
